Cahiers Société

\title{
Castoriadis et la critique de l'économie politique
}

\section{Maxime Ouellet}

Numéro 1, mai 2019

Castoriadis et les sciences sociales

URI : https://id.erudit.org/iderudit/1068425ar

DOI : https://doi.org/10.7202/1068425ar

Aller au sommaire du numéro

Éditeur(s)

Collectif Société

ISSN

2562-5373 (imprimé)

2562-5381 (numérique)

Découvrir la revue

Citer cet article

Ouellet, M. (2019). Castoriadis et la critique de l'économie politique. Cahiers Société, (1), 173-192. https://doi.org/10.7202/1068425ar

\section{Résumé de l'article}

Dans le cadre de cet article, nous analyserons le rapport qu'entretient Castoriadis avec la critique de l'économie politique élaborée par Marx et le marxisme. Nous voulons souligner que la théorie critique de la société capitaliste moderne formulée par Castoriadis demeure fortement inspirée par la pensée de Marx, pensée de laquelle il n’a jamais véritablement réussi à se libérer, et ce, en dépit de sa prétention à instituer une rupture définitive avec cette dernière. Cette première trame argumentative servira d'assise pour juger de la postérité de la pensée castoriadienne dans les sciences sociales contemporaines en général, et dans le domaine de l'économie politique et de la sociologie économique en particulier. Nous montrerons que certains éléments de sa pensée nourrissent la réflexion contemporaine en sociologie économique et en économie politique critique. Tant sa critique du marxisme traditionnel que sa réflexion sur le rôle des représentations dans l'orientation de l'agir économique trouvent écho dans certains travaux contemporains en économie politique critique qu'on pourrait qualifier de « post-marxistes ». 


\title{
Castoriadis et la critique de l'économie politique
}

\author{
Maxime OUELLET \\ Université du Québec à Montréal
}

Dans son «Bilan provisoire du marxisme » qui tient lieu de chapitre inaugural à son œuvre phare L'institution imaginaire de la société, Castoriadis énonce la phrase célèbre : «Partis du marxisme révolutionnaire, nous sommes arrivés au point où il fallait choisir entre rester marxistes et rester révolutionnaires ${ }^{1} »$. Dans ce texte, qu'il convient d'appeler, suivant Daniel Bensaïd, son "règlement de compte» avec le marxisme ainsi qu'avec son propre passé militant ${ }^{2}$, Castoriadis soutient que la pensée de Marx serait demeurée prisonnière des catégories de l'économie politique et n'aurait donc pas permis « de poser un projet politique alternatif axé sur la créativité humaine 3 ». Castoriadis reproche principalement à Marx son objectivisme scientiste - qu'on retrouve notamment au sein de la théorie de la valeur-travail - lequel serait au fond tout à fait compatible avec les postulats productivistes, utilitaristes et technicistes du capitalisme. Face à ce constat sans équivoque proposé par l'ancien militant trotskyste, il convient de se demander si Castoriadis ne serait pas qu'un énième représentant de la génération de philosophes marxistes français ayant cédé au chant des sirènes postmodernes 4 ?

Nous reviendrons donc dans le cadre de cet article sur le rapport qu'entretient Castoriadis avec la critique de l'économie politique élaborée par Marx et le marxisme. Il ne s'agit pas tant de proposer une analyse exhaustive du rapport critique qu'entretient Castoriadis avec Marx et le marxisme, exercice qui a déjà été effectué ailleurs 5 , que de montrer l'originalité de son analyse et sa pertinence pour la sociolo-

\footnotetext{
${ }^{1}$ Cornelius Castoriadis, L'institution imaginaire de la société, Paris, Seuil, 1999, p. 21.

2 Daniel Bensaïd, «Politiques de Castoriadis, Castoriadis corrige Marx », Contretemps, 26 septembre 2017. En ligne : http://www.contretemps.eu/politiques-de-castoriadis/ (consulté le 11 janvier 2018).

3 Nicolas Poirier, L'ontologie politique de Castoriadis, Paris, Payot, 2011 p. 185.

4 C'est du moins ce que semble soutenir la philosophe marxiste française Isabelle Garo dans sa critique de Castoriadis. Voir Isabelle Garo, «Imagination et représentation. De Castoriadis à Marx : rester marxistes ou rester révolutionnaires", Séminaire "Marxismes au XXI siècle». En ligne : http://semimarx.free.fr/IMG/pdf/GARO Castoriadis et Marx.pdf, séminaire Marx (consulté le 13 décembre 2016).

5 Voir entre autres Daniel Bensaïd, «Politiques de Castoriadis, Castoriadis corrige Marx », op. cit. ; Nicolas Poirier, L'ontologie politique de Castoriadis, op. cit. ; Isabelle Garo, « Imagination et représentation. De Castoriadis à Marx : Rester marxistes ou rester révolutionnaires », op. cit.
} 
gie contemporaine. Suivant l'analyse de Nicolas Poirier6, nous soutiendrons que les critiques de Castoriadis s'adressent davantage au marxisme qu'à Marx lui-même. Il ne s'agit pas d'argumenter contre Castoriadis qu'il existerait un vrai Marx caché derrière "l'ensemble des contre-sens qui a été fait en son nom7", mais plutôt de souligner la pertinence de la critique castoriadienne à l'endroit du «marxisme traditionnel ». Nous voulons toutefois souligner que la théorie critique de la société capitaliste moderne formulée par Castoriadis demeure malgré tout fortement inspirée par la pensée de Marx, pensée de laquelle il n'a jamais véritablement réussi à se libérer, et ce, en dépit de sa prétention à instituer une rupture définitive avec cette dernière. Cette première trame argumentative servira d'assise pour juger de la postérité de la pensée castoriadienne dans les sciences sociales contemporaines en général, et dans le domaine de l'économie politique et de la sociologie économique en particulier. En effet, bien que Castoriadis soit davantage considéré comme un théoricien de la philosophie politique que de l'économie politique ${ }^{8}$, nous montrerons que certains éléments de sa pensée nourrissent la réflexion contemporaine en sociologie économique et en économie politique critique. Tant sa critique du marxisme traditionnel que sa réflexion sur le rôle des représentations dans l'orientation de l'agir économique trouvent écho dans certains travaux contemporains en économie politique critique qu'on pourrait qualifier de « post-marxistes ».

Pour ce faire, il s'agira dans un premier temps d'exposer les similitudes entre la critique du «marxisme traditionnel» développée par le courant de la critique de la valeur9 et celle de Castoriadis. Nous présenterons d'abord schématiquement les principales critiques adressées par Castoriadis envers ce qu'il nomme le marxisme traditionnel pour ensuite présenter les éléments de sa critique de l'imaginaire capitaliste. Nous verrons que sa critique de la conception de la valeur comme substance, qui est au fondement de la théorie de la valeur-travail au sein du marxisme traditionnel, possède des similarités avec certaines relectures contemporaines de Marx, notamment celles développées par le marxisme critique de Lukács et de l'École de Francfort et, plus près de nous, dans la relecture critique de la théorie de la valeur proposée par Moishe Postone ${ }^{10}$. Ensuite, nous verrons que la critique du marxisme traditionnel développée par Castoriadis s'inscrit dans le contexte des transformations institutionnelles du capitalisme avancé, qu'il nomme capitalisme bureaucratique. Finalement, il s'agira de montrer comment la conception castoriadienne de l'imaginaire économique et de la valeur comme institution social-historique inspire un certain nombre

\footnotetext{
6 Nicolas Poirier, L'ontologie politique de Castoriadis, op. cit.

7 Michel Henry, Marx : une philosophie de la réalité, Paris, Gallimard, 1991, vol. 1, p. 9.

8 On oublie trop souvent qu'en plus d'avoir été philosophe et psychanalyste, Castoriadis a été économiste à l'OCDE de 1948 à 1970.

9 Pour un aperçu du courant de la critique de la valeur, voir Éric Martin et Maxime Ouellet (dir.), $L a$ tyrannie de la valeur. Débats pour le renouvellement de la théorie critique, Montréal, Écosociété, 2014. ${ }^{10}$ Moishe Postone, Temps, travail et domination sociale, Paris, Mille et une nuits, 2009.
} 
d'écrits post-marxistes qui cherchent à réactiver la critique de l'économie politique à l'ère du capitalisme financiarisé.

\section{Castoriadis et la critique du marxisme traditionnel}

La critique du marxisme élaborée par Castoriadis débute lorsqu'il militait au sein du groupe Socialisme ou Barbarie. Celle-ci vise principalement à remettre en question les postulats sur lesquels s'est édifié le marxisme traditionnel. Par «marxisme traditionnel », Castoriadis réfère à la lecture «objectiviste » de Marx qui était alors largement prédominante tant comme doctrine officielle des régimes du « socialisme réellement existant» que dans la lecture académique proposée par le marxisme dit « scientifique » dont le structuralisme althussérien représentait, du moins en France, la forme hégémonique. On peut résumer la critique du marxisme traditionnel formulée par Castoriadis en trois points. Premièrement, le marxisme traditionnel reproduit les failles des tendances positivistes et utilitaristes des économistes libéraux en consolidant une vision déterministe fondée sur le développement technique comme moteur de l'histoire. Ce développement se fonderait sur des lois objectives et scientifiques et ses contradictions mèneraient inévitablement à l'effondrement du capitalisme. Deuxièmement, l'approche traditionnelle transhistorise les catégories d'analyse comme la valeur, le travail, la marchandise, alors que celles-ci ne possèdent un sens selon Castoriadis que dans les sociétés capitalistes. Troisièmement, le marxisme traditionnel reproduit le postulat anthropologique libéral et essentialiste selon lequel il existerait une nature humaine inaltérable reposant uniquement sur la motivation économique ${ }^{11}$.

Ce type d'analyse transhistorique qui définit la dynamique objective des contradictions du capitalisme comme résultant du conflit entre le développement illimité des forces productives et les rapports de production a conduit le marxisme traditionnel à conclure que la suppression de la propriété privée et du marché conduirait logiquement à l'abolition du capitalisme. C'est à partir de l'exemple du socialisme réellement existant tel qu'il s'est légitimé en prenant appui sur les écrits de Marx, que Castoriadis en vient à la conclusion de l'invalidité des thèses marxistes. Comme il l'écrit dans un texte publié en 1959-1961 intitulé, «Le mouvement révolutionnaire sous le capitalisme moderne » :

La critique du capitalisme consiste finalement à dire qu'il ne développe pas assez vite les forces productives (ce qui revient à dire qu'il n'est pas assez capitalisme). Pour réaliser ce développement plus rapide, il faudrait et il suffirait que la propriété privée et le marché soient éliminés : nationalisation des moyens de produc-

${ }^{11}$ Cf. Cornelius Castoriadis, L'institution imaginaire de la société, op. cit., p. 42-43. 
tion et planification offriraient alors la solution à la crise de la société contemporaine ${ }^{12}$.

Si Castoriadis voit dans le marxisme traditionnel une idéologie qui sert de discours de légitimation à une forme particulière de capitalisme, c'est-à-dire le capitalisme bureaucratique d'État de type soviétique, il soutient également que les errances du marxisme reposent sur des antinomies qu'on retrouve au sein même de la pensée de Marx. Antinomie entre le germe révolutionnaire et antispéculatif - sur lequel Castoriadis prendra appui tout au long de son œuvre pour souligner l'aspect créatif de la praxis -, et ce qu'il considère comme l'élément théorique systématique, objectiviste et déterministe de Marx. C'est principalement cette dimension de la pensée de Marx qu'il critiquera tout au long de son œuvre.

Or, à regarder de plus près, on peut dire que la démarche de Castoriadis n'est pas si différente de la pensée de Marx, à condition bien sûr d'en expurger les antinomies qui peuvent effectivement apparaître dans son œuvre ${ }^{13}$. Sur le plan de la méthode, l'approche de Castoriadis s'apparente à la critique catégorielle opérée par Marx dans sa critique de l'économie politique. Dans un texte intitulé «La rationalité du capitalisme ", Castoriadis dit procéder à une critique immanente du capitalisme, c'est-àdire qu'elle

essaie de montrer que, sur le plan théorique, les constructions de l'économie politique académique sont incohérentes, ou privées de sens, valables seulement pour un monde fictif; et que, sur le plan empirique, le fonctionnement de l'économie capitaliste n'a que peu de rapport avec ce qui en est dit dans la théorie ${ }^{14}$.

Il s'agit donc selon Castoriadis de faire une critique du capitalisme selon ses propres critères. C'est également à partir de la même démarche que Moishe Postone propose sa relecture de Marx dans son ouvrage Temps, travail et domination sociale qui repose sur une critique du marxisme traditionnel s'apparentant à celle de Castoriadis ${ }^{15}$.

\footnotetext{
12 Cornelius Castoriadis, "Le mouvement révolutionnaire sous le capitalisme moderne », dans Capitalisme moderne et révolution, II. Le mouvement révolutionnaire dans le capitalisme moderne, Paris, Union générale d'éditions, 1979, p. 103-104.

13 Caumières souligne d'ailleurs que Castoriadis a utilisé les munitions théoriques du marxisme pour les retourner contre ce dernier : «Une des originalités de la critique de Castoriadis, qui apparaît ici clairement, consiste à emprunter au marxisme un de ses principes fondamentaux - qui veut que la connaissance historique est historique - pour le retourner contre le marxisme même ». Philippe Caumières, « Le social-historique chez Castoriadis et le poids de la pensée héritée », dans Cahiers Castoriadis, II. Imaginaire et création historique, dir. Philippe Caumières, Sophie Klimis et Laurent Van Eynde, Bruxelles, Facultés Universitaires Saint-Louis, 2006, p. 43.

14 Cornelius Castoriadis, «La rationalité du capitalisme », dans Les carrefours du labyrinthe, VI. Figures du pensable, Paris, Seuil, 1999, p. 83.

15 Pour une analyse des similitudes entre la critique du capitalisme de Postone et celle de Castoriadis, voir Bernard Pasobrola, «Fin du travail : version Postone ou Castoriadis », La Revue des ressources,
} 
En dépit de leur démarche similaire, la lecture proposée par Postone soutient cependant que la pensée de Marx ne serait pas aussi antinomique que le prétend Castoriadis. À cet égard, il faut se rappeler que, dans un texte célèbre intitulé "Valeur, égalité, justice, politique, de Marx à Aristote et d'Aristote à nous ${ }^{16}$ ", Castoriadis reprend le commentaire formulé par Marx à propos d'Aristote dans le premier chapitre du Capital17. Marx se pose la même question qu'Aristote dans l'Éthique à Nicomaque ${ }^{18}$, à savoir : comment est-il possible d'échanger deux choses qui sont par nature incommensurables ? À cette question, Aristote répond que l'échange n'est possible qu'à partir d'une convention relevant de «l'œuvre du nomos, de la loi, de l'institution social-historique ${ }^{19} »$. Selon Marx, si Aristote pose la bonne question, il ne peut trouver une réponse adéquate à celle-ci puisque la société grecque qui reposait sur l'esclavagisme n'avait pas encore institué la norme du temps de travail abstrait comme fondement de la valeur. Aussi affirme-t-il :

Mais ce qu'Aristote ne pouvait pas lire dans la forme-valeur proprement dite, c'est que, sous la forme des valeurs marchandes, tous les travaux sont exprimés comme du travail humain égal, comme du travail valant donc la même chose, et cela parce que la société grecque reposait sur le travail des esclaves, et qu'elle avait donc comme base naturelle l'inégalité des hommes et de leurs forces de travail. Le secret de l'expression de valeur, l'égalité et l'égale validité de tous les travaux parce que et pour autant que ceux-ci sont du travail humain en général, ne peut être déchiffré qu'à partir du moment où le concept d'égalité humaine a acquis la solidité d'un préjugé populaire. Or ceci n'est possible que dans une société où la forme-marchandise est la forme générale des produits du travail, et où donc également c'est le rapport entre les hommes en tant que possesseurs de marchandises qui est le rapport social dominant. Tout le génie d'Aristote éclate précisément dans le fait qu'il découvre un rapport d'égalité au sein de l'expression de valeur des marchandises. C'est seulement l'obstacle historique de la société dans laquelle il vivait, qui l'empêche de déceler en quoi consiste «en vérité » ce rapport d'égalité2o.

vendredi 2 octobre 2009. En ligne : http://www.larevuedesressources.org/fin-du-travail-version-postoneou-castoriadis, $1290 . \mathrm{html}$.

16 Cornelius Castoriadis, "Valeur, égalité, justice, politique, de Marx à Aristote et d'Aristote à nous ", dans Les Carrefours du labyrinthe, I, Paris, Seuil, 1978, p. 325-413.

17 Karl Marx, Le Capital, Livre I, Paris, Presses Universitaires de France, 2006.

18 Aristote, Éthique de Nicomaque, Paris, Garnier-Flammarion, 1965.

19 Cornelius Castoriadis, "Valeur, égalité, justice, politique, de Marx à Aristote et d'Aristote à nous », dans Les carrefours du labyrinthe, I, op. cit., p. 350.

${ }^{20}$ Karl Marx, Le Capital, op. cit, p. 68. 
C'est pourquoi Castoriadis soutiendra que Marx se fait métaphysicien en posant le travail comme substance transhistorique de la valeur. Pour Postone, cette conception substantialiste doit plutôt être comprise comme un métacommentaire portant sur les formes fétichisées de pensée qui sont propres aux sociétés capitalistes. En ce sens, la catégorie de la valeur-substance permettrait de comprendre comment Marx saisit la valeur à la lumière de la catégorie hégélienne du Sujet. La valeur qui s'autovalorise, constituée par la médiation des rapports sociaux au moyen du travail abstrait, serait le Sujet du capitalisme au sens hégélien du terme ; il s'agit d'un "sujet automate ». Alors que Castoriadis perçoit la conception substantialiste comme un voile empêchant de saisir la nature historique de la valeur, Postone y voit un moyen de comprendre le fonctionnement effectif du capitalisme. Bref, selon Postone, ce ne serait pas Marx qui serait métaphysicien, comme le soutient Castoriadis, ce serait plutôt le Capital qui fonctionnerait de manière métaphysique en ce que ses rapports sociaux constitutifs se présenteraient sous la forme d'une entité naturelle et transhistorique ${ }^{21}$.

Castoriadis reproche en effet à Marx de dire une chose et son contraire au sein d'un même chapitre, soit de défendre, d'un côté, une conception social-historique de la valeur qui repose sur la spécificité historique des rapports sociaux médiatisés par la marchandise et le travail abstrait et, d'un autre côté, une conception substantialiste qui ferait du travail une essence transhistorique au fondement de la richesse dans toutes les sociétés à travers l'histoire. Or comme le souligne Postone :

[Castoriadis] oublie la nature immanente de la critique de Marx lorsqu'il affirme qu'elle est métaphysique et implique une ontologification du travail. Castoriadis lit implicitement la critique négative de Marx comme une science positive et il la critique donc sur cette base; il n'examine pas la relation entre l'analyse catégorielle de Marx et le concept de fétiche-marchandise, et prête à Marx un incroyable degré d'incohérence ${ }^{22}$.

\footnotetext{
${ }^{21}$ Selon Pierre Dardot, malgré leurs divergences, Castoriadis et Postone en arrivent à la même conclusion, mais par des voies opposées. Ils se tromperaient toutefois tous les deux puisque Marx n'utiliserait pas le concept de substance dans un sens métaphysique dans le premier chapitre du Capital. Son concept de substance référerait à une " substance sociale " et non à une " substance-essence ", qui, elle, serait métaphysique. Dardot clôt le débat en donnant une inflexion plutôt foucaldienne à l'analyse de Marx, en disant que plutôt que de voir le capitalisme comme une institution au sens castoriadien du terme, il serait préférable d'y donner un sens plus sobre, c'est-à-dire «celui de règles ou de normes dont la mise en place ne procède pas de "conventions" et dont la logique est de produire des sujets qui se rapportent à eux-mêmes comme à des valeurs à valoriser “toujours plus" ». Pierre Dardot, " La valeur n'est pas une substance », dans La tyrannie de la valeur. Débats pour le renouvellement de la théorie critique, dir. Éric Martin et Maxime Ouellet, op. cit., p. 144.

22 Moishe Postone, Temps, travail et domination sociale, op. cit., p. 254.
} 


\section{Castoriadis et la critique de l'imaginaire capitaliste}

Pourtant, si l'on suit l'analyse proposée par Castoriadis dans son œuvre majeure, L'institution imaginaire de la société, force est de constater qu'elle prend appui sur deux concepts marxiens fondamentaux, ceux de fétichisme et d'aliénation. Ces catégories ont effectivement été éliminées du vocabulaire du marxisme traditionnel sous prétexte qu'il s'agissait là de coquetteries hégéliennes - pour reprendre les termes d'Althusser - relevant de résidus d'une pensée non scientifique qu'il était nécessaire de surpasser au profit d'une réelle «science marxiste». Castoriadis souligne d'ailleurs que lorsque Marx « parlait du fétichisme de la marchandise, et montrait son importance pour le fonctionnement effectif de l'économie capitaliste, il dépassait de toute évidence la vue simplement économique et reconnaissait le rôle de l'imaginaire 23 ». En ce sens, on peut dire que Castoriadis rejoint en partie la relecture de Marx proposée par Postone, pour qui les formes de conscience humaine, tout comme les formes objectives que prennent les rapports sociaux, sont des éléments médiatisés par la totalité, c'est-à-dire par l'institution symbolique du social. Cette lecture rejette explicitement l'analyse déterministe du marxisme traditionnel selon laquelle la base matérielle déterminerait la superstructure symbolique, politique et institutionnelle, au profit d'une analyse des médiations sociales constitutives des formes d'objectivités et de subjectivités sociales, donc des formes de conscience humaine. Comme le dit également Castoriadis :

Il serait finalement moins faux de dire que l'homo oconomicus est un produit de la culture capitaliste que de dire que la culture capitaliste est une création de l'homo æeconomicus. Mais il ne faut dire ni l'un ni l'autre. Il y a chaque fois homologie et correspondance profonde entre la structure de la personnalité et le contenu de la culture, et il n'y a pas de sens à prédéterminer l'une par l'autre24.

Suivant cette lecture, la critique de l'économie politique doit donc tenir compte des modes d'abstraction et d'objectivation propres à un contexte social-historique donné. Par exemple, la théorie de la valeur-travail est la forme de représentation symbolique, culturelle et normative accordée au travail, en tant que forme objectivée de médiation sociale spécifique aux sociétés capitalistes. Ce type de médiation sociale est inintelligible dans les sociétés antérieures; le sujet ne pouvait y être imaginé comme une chose mesurable en temps de travail et transformable en force productive, c'est-à-dire sous une forme réifiée. L'analyse de ce type de médiation doit donc considérer le moment historique dans lequel ce nouveau sens accordé à l'activité

23 Cornelius Castoriadis, L'institution imaginaire de la société, op. cit., p. 198.

24 Ibid., p. 41. 
sociale comprise comme «travail » a été institué en tant que fondement de l'ordre symbolique ${ }^{25}$. À cet égard, Castoriadis souligne que :

Un esclave n'est pas un animal, un ouvrier n'est pas une chose ; mais la réification n'est ni une fausse perception, ni une erreur logique $[\ldots]$. La réification est une signification imaginaire (inutile de souligner que l'imaginaire social, tel que nous l'entendons, est plus réel, que le « réel »). Du point de vue étroitement symbolique, ou « linguistique », elle apparait comme un déplacement de sens, comme une combinaison de métaphore et de métonymie. L'esclave ne peut «être » animal que métaphoriquement, et cette métaphore, comme toute métaphore, s'appuie sur une métonymie, la partie étant prise pour le tout aussi bien chez l'animal que chez l'esclave et la pseudo-identité des propriétés partielles étant étendue sur le tout des objets considérés. Mais ce glissement de sens qui est après tout l'opération indéfiniment répétée du symbolisme - , le fait que sous un signifiant survient un autre signifié, est simplement une façon de décrire ce qui s'est passé, et ne rend compte ni de la genèse, ni du mode d'être du phénomène. Ce dont il s'agit dans la réification - dans le cas de l'esclavage ou dans le cas du prolétariat -, c'est l'instauration d'une nouvelle signification opérante, la saisie d'une catégorie d'hommes par une autre catégorie comme assimilable, à tous égards pratiques, à des animaux ou à des choses ${ }^{26}$.

D'ailleurs, selon Castoriadis, l'imaginaire de l'institution social-historique du capitalisme repose sur une forme de réification qui consiste à remplacer des valeurs sociales par le critère économique. L'économie réifiée s'érige ainsi comme «valeur suprême de la vie sociale 27 » :

L'économie au sens le plus large (de la production à la consommation) passe pour l'expression par excellence de la rationalité du capitalisme et des sociétés modernes. Mais c'est l'économie qui exhibe de la façon la plus frappante - précisément parce qu'elle se prétend intégralement exhaustivement rationnelle - la domination de l'imaginaire à tous les niveaux ${ }^{28}$.

\footnotetext{
25 Éric Martin et Maxime Ouellet, «Introduction: la crise du capitalisme est aussi la crise de l'anticapitalisme », dans La tyrannie de la valeur. Débats pour le renouvellement de la théorie critique, op. cit., p. 20.

${ }^{26}$ Cornelius Castoriadis, L'institution imaginaire de la société, op. cit., p. 212-213.

27 Cornelius Castoriadis, "La rationalité du capitalisme», dans Les carrefours du labyrinthe, VI, op. cit., p. 84.

28 Cornelius Castoriadis, L'institution imaginaire de la société, op. cit., p. 236.
} 
C'est en ce sens que Castoriadis reprendra le concept d'aliénation développé par le jeune Marx afin de montrer que la spécificité de la domination sociale propre au capitalisme ne peut se comprendre en dehors de sa fonction symbolique. Il définit l'aliénation comme l'autonomisation et la domination des moments imaginaires dans l'institution, " qui entraîne[nt] l'autonomisation et la domination de l'institution relativement à la société29 ». Dans un contexte d'aliénation, la société ne reconnaît plus l'imaginaire et son institutionnalisation comme son propre produit. En ce sens, l'autonomisation de la sphère économique induit une domination abstraite qui s'exerce sous la forme d'une contrainte dépersonnalisée et rationnelle que l'on ne peut comprendre uniquement comme une domination concrète exercée soit par des groupes sociaux, des classes, ou des institutions économiques et politiques. Comme le souligne Castoriadis : "dans une société d'aliénation la classe dominante ellemême est en situation d'aliénation [...], elle ne peut mystifier le reste de la société avec son idéologie sans se mystifier en même temps elle-même ${ }^{30}$ ».

L'analyse de l'imaginaire dans le capitalisme conduit donc Castoriadis à rejeter le schéma dualiste base/superstructure au profit d'une analyse des transformations symboliques et politico-institutionnelles qu'il présente comme des conditions historiques de possibilité du capitalisme. On comprend donc, suivant Castoriadis, que les conditions de possibilité du capitalisme reposent sur des critères non matériels qui n'ont rien à voir avec l'économique. Il en énumère une série :

1. Éclosion scientifique qui commence déjà avant la Renaissance. Son analyse suit en cela celles de Lukács et de l'École de Francfort, pour qui la raison moderne s'est édifiée sur l'expulsion du moment subjectif et sensible de la pratique humaine, ce qui a eu pour conséquence qu'elle s'est présentée sous la forme universelle et abstraite de la quantification ${ }^{31}$. Cette mathématisation du réel s'est transformée selon Castoriadis dans la période récente en mouvement autonome de la technoscience menant à l'élimination du rôle de l'homme dans la production.

2. Naissance et consolidation de l'État moderne et de sa bureaucratisation qui fournira un modèle d'organisation à l'entreprise moderne.

\footnotetext{
29 Ibid., p. 198.

30 Ibid., p. 171.

${ }^{31}$ Castoriadis s'appuie explicitement sur la théorie de la réification de Lukács pour développer sa thèse portant sur l'imaginaire dans les sociétés capitalistes mais se distancie du déterminisme téléologique qu'on retrouve dans l'hégéliano-marxisme. Si sa critique de la rationalité instrumentale se rapproche de la première génération de l'École de Francfort (Adorno, Horkheimer, Marcuse), il ne dialogue pratiquement jamais avec ces derniers. Le principal point commun qu'on peut identifier entre leurs approches respectives est de produire une critique de la raison instrumentale qui ne cherche pas à sacrifier pour autant ce qui dans le raison conserve un caractère émancipateur. À ce sujet, voir Nicolas Poirier, L'ontologie politique de Castoriadis, op. cit., p. 29-30.
} 
3. Formation des nations modernes qui, du point de vue économique et politique, permet l'unification du marché ainsi que la mise en place de règles essentielles à la première phase du développement du capitalisme.

4. Mutation anthropologique qui fait de l'être humain un homo æeconomicus.

5. Finalement, et surtout, le capitalisme naît dans une société où le conflit est présent dès le départ, c'est-à-dire où la mise en question de l'ordre établi est également constitutive de l'institution symbolique du social ${ }^{2}$.

Ce dernier constat amènera Castoriadis à postuler, dans une optique hyperhégélienne, que seule l'époque moderne est réellement animée par une contradiction dialectique. Pour Castoriadis, la contradiction du capitalisme n'est pas économique, mais bien politique : «la société capitaliste est la première société historique que nous connaissons dont l'organisation contienne une contradiction interne insurmontable 33 ». En effet, selon lui, bien qu'il existe des classes sociales dans les sociétés précapitalistes, ceci ne veut pas dire pour autant qu'il existe une contradiction au fondement de ces formations sociales. Il n'y aurait donc pas de dialectique possible entre le maître et l'esclave au sens hégélien dans les sociétés précapitalistes, puisque maître et esclave ne partagent pas un monde commun : « ce n'est pas l'activité quotidienne des exploités qui oblige quotidiennement les exploiteurs à transformer leur société34 ». Seule la société capitaliste a institué un imaginaire social qui rend possible la contradiction dialectique :

Le capitalisme au contraire, est bâti sur une contradiction intrinsèque - une contradiction vraie, au sens littéral du terme. L'organisation capitaliste de la société est contradictoire au sens rigoureux où l'individu névrosé l'est : elle ne peut tenter de réaliser ses intentions que par des actes qui les contrarient constamment. Pour se situer au niveau fondamental, celui de la production: le système capitaliste ne peut vivre qu'en essayant continuellement de réduire les salariés en purs exécutants - et il ne peut fonctionner que dans la mesure où cette réduction ne se réalise pas : le capitalisme est obligé de solliciter constamment la participation des salariés au processus de production, participation qu'il tend par ailleurs lui-même à rendre impossible. Cette même contradiction se retrouve, en termes presque identiques, dans les domaines de la politique ou de la culture35.

32 Cornelius Castoriadis, «La rationalité du capitalisme», dans Les carrefours du labyrinthe, VI, op. cit., p. 90-91.

33 Cornelius Castoriadis, "Le mouvement révolutionnaire sous le capitalisme moderne », op. cit., p. 105.

34 Ibid., p. 106

35 Ibid., p. 106-107. 
Pour Castoriadis, la contradiction du capitalisme n'a rien à voir avec la soi-disant loi objective de la baisse tendancielle du taux de profit. Il s'agirait plutôt d'une contradiction intrinsèquement politique, qu'il analyse d'abord au sein de l'usine dans ses écrits de l'époque de Socialisme ou Barbarie et qu'il étendra par la suite à l'ensemble de la société capitaliste moderne. Dans ses "écrits de jeunesse », la contradiction fondamentale du capitalisme ne porte pas tant sur la question de la propriété des moyens de production que sur l'opposition entre dirigeants et dirigés. Plus tard, Castoriadis reformulera cette contradiction à partir de son travail de théorisation portant sur l'imaginaire des sociétés modernes ${ }^{36}$. Deux significations imaginaires antinomiques seraient au fondement des sociétés modernes : d'une part, la volonté d'autonomie qui anime tout projet d'émancipation politique fondé sur l'agir réflexif en commun et, d'autre part, l'imaginaire capitaliste fondé sur la domination irréfléchie de la nature en vue de la croissance illimitée :

L'Occident moderne, depuis des siècles, est animé par deux significations imaginaires sociales tout à fait opposées, même si elles se sont contaminées réciproquement : le projet d'autonomie individuelle et collective, la lutte pour l'émancipation de l'être humain, aussi bien intellectuelle et spirituelle qu'effective dans la réalité sociale ; et le projet capitaliste, démentiel, d'une expansion illimitée d'une pseudo-maîtrise pseudo-rationnelle, qui depuis longtemps a cessé de concerner seulement les forces productives et l'économie pour devenir un projet global (et pour autant encore plus monstrueux), d'une maîtrise totale des données physiques, biologiques, psychiques, sociales, culturelles. Le totalitarisme n'est que la pointe la plus extrême de ce projet de domination qui du reste s'inverse dans sa propre contradiction, puisque même la rationalité restreinte, instrumentale du capitalisme classique devient chez lui irrationalité et absurdité, comme le stalinisme et le nazisme l'ont montré37.

En effet, selon Castoriadis, l'analyse de la contradiction entre forces productives et rapports de production laisse intacte la critique de la rationalité du capitalisme telle qu'elle s'incarne plus spécifiquement dans le développement de la technique moder-

\footnotetext{
${ }^{36}$ Si Gilles Labelle soutient qu'on ne peut pas séparer l'œuvre de Claude Lefort en deux temps, à savoir l'époque associée à Socialisme ou Barbarie et l'époque post-marxiste, on peut dire qu'il en va de même pour Castoriadis. Voir Gilles Labelle, "Parcours de Claude Lefort : de 1'“expérience prolétarienne" de 1'“aliénation" à la critique du marxisme », Politique et Sociétés, vol. 34, n 1, 2015, p. 17-36. Pour une comparaison entre la pensée de Lefort et Castoriadis voir, dans le même numéro, Antoine Chollet, « Claude Lefort et Cornelius Castoriadis : regards croisés sur Mai 68 », Politique et Sociétés, op. cit., p. 37-60.

37 Cornelius Castoriadis, «La montée de l'insignifiance », dans Les carrefours du labyrinthe, IV. La montée de l'insignifiance, Paris, Seuil, 1996, p. 105-106.
} 
ne38. La spécificité de la société capitaliste par rapport aux sociétés antérieures est justement qu'elle repose sur une forme de domination qui se légitime par la Raison alors que les autres formes d'institution de la société se légitimaient à partir d'éléments mythiques ou religieux. Selon Castoriadis, ce qui caractérise la société capitaliste, c'est l'extension illimitée de la maîtrise rationnelle qui n'est pas seulement orientée vers la conquête extérieure - comme dans l'analyse marxiste classique de l'impérialisme -, mais qui vise également la totalité de la société. Il s'agit d'une extension du contrôle qui repose sur la domination de la rationalité instrumentale à l'ensemble des sphères de la pratique sociale, pour reprendre le vocabulaire de l'École de Francfort. C'est sur ce point précis que l'analyse castoriadienne montre la limite principale de l'analyse marxienne et marxiste. Celle-ci est ancrée dans le contexte du capitalisme libéral du XIX ${ }^{\mathrm{e}}$ siècle qui demeurait dominé par les rapports sociaux de type bourgeois, c'est-à-dire où la rationalité de la valeur n'avait pas colonisé l'entièreté de l'espace social et culturel. Dans la lignée des premiers écrits de Lukács portant sur la réification et de l'École de Francfort, la critique de Castoriadis tentera de saisir la spécificité de la domination propre au capitalisme avancé, qu'il désignera par le terme de " capitalisme bureaucratique », au sein duquel la médiation des rapports sociaux au moyen du marché se substitue à un contrôle exercé sur l'ensemble de la vie sociale par les organisations.

\section{La critique du capitalisme bureaucratique}

La critique du capitalisme bureaucratique proposée par Castoriadis consiste en une synthèse originale entre les théoriciens classiques en sociologie (Marx, Weber et Durkeim) et les travaux post-keynésiens associés à l'École de Cambridge (Straffa, Robinson, Kalecki, Schakle, Kaldor, Pasinetti). De Marx, on l'a vu, il retiendra en partie sa théorie de l'aliénation et du fétichisme de la marchandise, de Weber, la critique de la rationalisation propre aux sociétés modernes, et de Durkheim, une analyse institutionnelle des faits économiques comme relevant des représentations collectives. De cette synthèse naîtra une critique de l'économie politique qui rejette la prétendue naturalité de l'économie au profit d'une analyse de l'institution économique comprise comme élément d'organisation du comportement des agents économiques. Il prolonge de la sorte à l'organisation bureaucratique l'analyse du rôle de l'imaginaire dans l'économie capitaliste telle qu'on la retrouve dans la conception marxienne du fétichisme de la marchandise. Pour Castoriadis, c'est l'organisation qui vient remplacer le marché comme forme institutionnelle prédominante au sein du

${ }^{8}$ Cf. Cornelius Castoriadis, «La rationalité du capitalisme», dans Les carrefours du labyrinthe, VI, op. cit. 
capitalisme avancé39. Dans le capitalisme avancé, l'organisation a désormais pour fonction de produire les significations qui font tenir ensemble la société :

L'univers bureaucratique est peuplé d'imaginaire d'un bout à l'autre [...]. Mais il y a bel et bien un système de significations imaginaires « positives » qui articulent l'univers bureaucratique, système que l'on peut reconstituer à partir des fragments et des indices qu'offrent les instructions sur l'organisation de la production et du travail, le modèle même de cette organisation, les objectifs qu'elle se propose, le comportement typique de la bureaucratie, etc. 40

L'imaginaire bureaucratique correspond à ce que Boltanski et Chiapello ont nommé à la suite de Weber un "esprit du capitalisme ». Cet imaginaire ne peut se réduire en un simple outil de manipulation idéologique de la part d'une nouvelle classe dirigeante, la bureaucratie, puisqu'il oriente également normativement la pratique des acteurs au sein de l'organisation. Il s'agit, pour reprendre les termes de Boltanski et Chiapello, d'

une idéologie qui justifie l'engagement des gens dans le capitalisme et qui rend ces engagements attrayants, en dépit du fait que le capitalisme constitue un système absurde qui oblige la subordination des travailleurs et enchaîne les capitalistes eux-mêmes dans un processus d'accumulation sans fin ${ }^{41}$.

Si Castoriadis est conscient de cet aspect normatif de l'imaginaire bureaucratique dans l'orientation significative de la pratique sociale, il n'abandonne pas pour autant l'analyse critique des effets aliénants de l'organisation capitaliste. Suivant Freitag, il serait peut-être préférable de parler d' « organisation imaginaire de la société42 » plutôt que d'institution, puisque le monde bureaucratique réalise à l'extrême l'autonomisation du pur symbolisme selon Castoriadis. Il autonomise la raison dans un de ses moments partiels, celui de l'entendement, et est indifférent par rapport aux fins ${ }^{43}$. Le capitalisme bureaucratique se caractérise notamment par une transformation de l'imaginaire de l'organisation qui passe d'une bureaucratie essentiellement conservatrice dont la finalité consiste à préserver les normes, la neutralité et la conti-

39 Pour une analyse sociologique du rôle de la corporation comme forme institutionnelle prédominante au sein du capitalisme avancé, voir François L'Italien, Béhémoth Capital. Genèse, développement et financiarisation de la grande corporation, Montréal, Nota Bene, 2016.

$4^{\circ}$ Cornelius Castoriadis, L'institution imaginaire de la société, op. cit., p. 239.

${ }^{41}$ Luc Boltanski et Ève Chapiello, Le nouvel esprit du capitalisme, Paris, Gallimard, 1999, p. 41.

42 Castoriadis, au contraire de Freitag, ne distingue pas conceptuellement la notion d'institution de celle d'organisation. Chez Freitag, l'institution s'appuie sur des finalités normatives transcendantes alors que l'organisation ne se légitime qu'à partir de la raison instrumentale. Dans le monde organisation, les moyens supplantent les fins. Cf. Michel Freitag, Le naufrage de l'université et autres essais d'épistémologie politique, Montréal, Nuit Blanche Éditions, 1995.

43 Cf. Cornelius Castoriadis, L'institution imaginaire de la société, op. cit., p. 240. 
nuité, à une organisation qui vise «l'anticipation systématique de l'avenir : le fantasme de l'organisation comme machine bien huilée cède la place », dit-il, « au fantasme de l'organisation comme machine autoréformatrice et autoexpensive44 ». Dans le capitalisme bureaucratique, le rôle de l'État est d'agir comme un "capitaliste global » dans la mesure où son intervention dans l'économie vise à « assurer la croissance économique dans la stabilité45 » au moyen du « développement de la consommation et des loisirs [, de] l'élargissement de l'éducation et [de] la diffusion de la culture ${ }^{46} »$. Non seulement la production, l'État et la politique ont-ils été bureaucratisés, mais également la consommation. La bureaucratisation de la consommation, sous la forme de services des ventes, de publicité et de la recherche de marchés, vise à trouver un débouché pour la logique d'expansion de la production. L'idéologie du libre choix du consommateur a cédé sa place à la «manipulation» des industries culturelles et du marketing afin de s'assurer de produire une culture adaptée à la logique de surproduction du capitalisme bureaucratique 47 . La bureaucratisation de la société comme forme institutionnelle prédominante trouve sa contrepartie subjective dans la privatisation de l'individu, c'est-à-dire dans la dépolitisation généralisée de l'ensemble des couches de la société, et plus particulièrement du prolétariat dont l'intégration dans la culture de masse a dissous sa conscience de classe au sein d'un gigantesque potlatch consumériste ${ }^{48}$.

Il reste que l'analyse du capitalisme bureaucratique élaborée par Castoriadis s'applique principalement à la configuration institutionnelle propre à l'époque fordiste où, pour la majorité des théoriciens critiques, l'intervention de l'État dans l'économie rendait la possibilité d'une crise structurelle du capitalisme inenvisageable. Castoriadis soulignait en 1963 qu' « une dépression du genre de celle de 1929 est désormais inconcevable, en dehors d'une crise de la folie collective des capitalistes $49 »$. Dans ce contexte, Castoriadis, comme la plupart des théoriciens critiques de l'époque, notamment ceux de l'École de Francfort, délaissera l'analyse des contradictions économiques propres au capitalisme avancé au profit de l'analyse des contradictions culturelles du capitalisme. Comme l'explique le sociologue allemand Wolgang Streeck, dans les années 1960-1970, pour la théorie critique s'inscrivant dans la lignée du marxisme occidental5o :

la faille du capitalisme ne résidait plus simplement dans son économie, mais dans sa politique, et dans sa société : non pas du côté de l'économie, mais dans la démocratie ; non pas du côté du capi-

\footnotetext{
44 Idem.

45 Cornelius Castoriadis, "Le mouvement révolutionnaire sous le capitalisme moderne », op. cit., p. 126.

46 Idem.

47 Ibid., p. 127-144.

48 Idem.

49 Ibid., p. 93.

50 Cf. Maurice Merleau-Ponty, Les aventures de la dialectique, Paris, Gallimard, 1955.
} 
tal, mais du travail [...]. Le problème désormais n'était plus la production de la plus-value - ses contradictions étaient devenues, pensait-on maîtrisables mais bien la légitimité du capital - non plus ce qu'un capital transformé pourrait fournir à la société, mais s'il pourrait fournir assez pour inciter cette société à continuer de jouer le jeu. La crise du capitalisme dans les années 1960-1970, n'était absolument pas pour cette raison une crise de la (sur- ou sous-) production, mais une crise de légitimation ${ }^{51}$.

Tout comme Daniel Bell52 qui interrogeait, dans une optique « néoconservatrice », la capacité des sociétés capitalistes à se reproduire dans un contexte où l'ascétisme propre à l'esprit du capitalisme est dévalorisé par la culture de consommation de masse, Castoriadis analysait, à partir d'une perspective "révolutionnaire ", les contradictions culturelles du capitalisme comme une crise des significations imaginaires qui permettent de tenir la société ensemble :

Qu'est-ce qui, dans la logique du capitalisme (ou de l'individualisme démocratique contemporain), interdit à un inspecteur des impôts de recevoir des pots-de-vin ? Pourquoi un enseignant devrait-il s'embêter à apprendre quelque chose s'il peut s'arranger avec son inspecteur? Un mathématicien de premier ordre, professeur en faculté, gagne peut-être 16000 francs par mois et produit des jeunes mathématiciens. Parmi ceux-ci, ceux qui savent ce qui se passe dans la vie (c'est-à-dire presque tous) ne continueront pas à faire des mathématiques; ils feront de l'informatique et entreront dans une firme avec un salaire au départ de peut-être 30000 francs. Qui donc, à la génération suivante, deviendra professeur de mathématiques? D'après la logique du système, à peu près personne. On dira : il y aura toujours de doux cinglés, qui aiment une belle démonstration plus qu'un salaire élevé. Mais je dis que, précisément, d'après les normes du système, de telles personnes ne doivent pas exister ; leur survivance est une anomalie systémique - de même que celle d'ouvriers consciencieux, de juges intègres, de bureaucrates wébériens, etc. Mais combien de temps un système peut-il se reproduire uniquement en fonction d'anomalies systémiques53 ?

Les transformations contemporaines du capitalisme ont montré la puissante plasticité et la résilience de cette forme sociale à surmonter ses propres contradictions, qu'elles soient économiques ou culturelles. En effet, les nouvelles théories managériales ont

${ }^{11}$ Wolgang Streeck, Du temps acheté. La crise sans cesse ajourné du capitalisme démocratique, Paris, Gallimard, 2014, p. 25.

$5^{2} C f$. Daniel Bell, The cultural contradictions of capitalism, New York, Basic Books, 1976.

53 Cornelius Castoriadis, «Quelle démocratie?», dans Les carrefours du labyrinthe, VI. Figures du pensable, Paris, Seuil, 1999, p. 213. 
récupéré la critique artistique qui dénonçait la culture massifiée du capitalisme bureaucratique pour produire un nouvel esprit du capitalisme fondé sur la notion d'autonomie individuelle54. Si le contexte social-historique de l'époque peut expliquer la substitution de l'intérêt que portait Castoriadis envers la critique de l'économie politique au profit d'une philosophie politique axée sur le concept d'autonomie, il n'en demeure pas moins que ses analyses serviront d'inspiration à certains auteurs contemporains qui aspirent à produire une théorie critique hétérodoxe de l'économie politique cherchant à dépasser les apories opposant les analyses subjectivistes et objectivistes dans le contexte de la montée en puissance du capitalisme financier.

\section{Théorisations contemporaines du capitalisme financier : l'apport de Castoriadis}

Castoriadis a peu écrit sur la montée en puissance du capitalisme financier. Dans un de ses derniers écrits datant de 1997, "La rationalité du capitalisme », terminé peu avant sa mort, il soutient tout de même que la montée du néolibéralisme consiste en la crise du projet social-historique d'autonomie individuelle et collective dont les bénéficiaires principaux sont les grandes firmes de l'industrie et de la finance55. Ce triomphe de l'imaginaire capitaliste se matérialise selon lui dans le démantèlement du rôle de l'État dans l'économie, et dans une régression idéologique « où la critique de l'économie politique académique telle que développée par l'École de Cambridge n'est plus discutée et même passée sous silence 56 ». Ce texte, qui synthétise ce qu'on pourrait appeler la sociologie économique de Castoriadis, propose une critique immanente du capitalisme qui vise à remettre en question les fondements de l'économie politique traditionnelle désormais dominée par le courant néoclassique. Selon Castoriadis, le capitalisme résulte d'un ensemble de conventions/institutions dont l'institution juridique de la propriété privée est l'instance principale. À partir de cette approche institutionnaliste, il démontre le caractère fictif de la rationalité du postulat fondateur de l'économie, à savoir la figure de l'homo æeconomicus qui vise à maximiser/minimiser tout instant et toute action. C'est donc sur la convention/institution juridique de la propriété que la théorie économique néoclassique dominante dans le monde académique est en mesure de mathématiser le réel. L'économétrie repose donc sur la fiction de l'imputation séparée qui surplombe l'ensemble de son raisonnement. En effet, selon Castoriadis, il est pratiquement impossible d'imputer rationnellement un résultat économique à tel ou tel facteur productif, que ce soit le capital ou le travail. La richesse ne peut s'approprier privément et être séparée à des fins de

54 Cf. Luc Boltanski et Ève Chiapello, Le nouvel esprit du capitalisme, op. cit.

55 Cornelius Castoriadis, «La rationalité du capitalisme», dans Les carrefours du labyrinthe, VI, op. cit., p. 110.

56 Ibid., p. 79. 
quantification qu'en faisant abstraction de son caractère social et historique. Ce n'est que par le biais de conventions qu'il est possible de le faire. Ainsi les conventions d'imputation et de séparation qui permettent la quantification et la mathématisation $\mathrm{du}$ réel ne font que nourrir tautologiquement la fiction du discours économique57. L'économie au sens moderne du terme est donc une invention, pour reprendre le titre du livre du penseur de la décroissance Serge Latouche, qui applique la conception castoriadienne de l'imaginaire à l'histoire de la pensée économique 58 .

Le concept d'imaginaire économique introduit par Castoriadis trouvera écho dans plusieurs travaux contemporains en économie politique critique à la suite du tournant « culturel » en sciences sociales dans les années 1980-1990. Il est notamment mobilisé dans le monde anglo-saxon au sein des approches d'économie politique culturelle. On le retrouve dans les travaux de Bob Jessop qui, dans une optique davantage gramscienne, s'appuie sur le concept d'imaginaire afin de comprendre la mobilisation d'un nouveau discours axé sur l'économie du savoir comme stratégie d'intégration des États-nations au sein du capitalisme globalisé59. La notion d'imaginaire économique est aussi utilisée dans certains travaux portant sur le rôle des zones offshore dans le contexte de la globalisation ${ }^{60}$.

C'est également en s'appuyant sur la critique castoriadienne de la théorie substantialiste de la valeur, défendue à la fois par l'école marginaliste de la valeur-utilité et les théoriciens marxistes traditionnels de la valeur-travail, qu'André Orléan construit sa critique de la valeur dans le capitalisme financier dans son ouvrage important intitulé : L'empire de la valeur ${ }^{61}$. Orléan soutient que la valeur est une institution socialhistorique qui ne correspond pas à la description qu'en donne l'école néoclassique ni le marxisme traditionnel. En essentialisant le travail ou l'utilité, les approches substantialistes de la valeur ne prennent pas en considération les représentations et les institutions sociales qui sont constitutives des normes et des pratiques au fondement de la valorisation économique. Dans cette optique, la valeur n'est pas une substance ; il s'agit plutôt d'un imaginaire social qui s'institutionnalise dans une période sociohistorique déterminée. Il ne s'agit de rien de moins pour Orléan que de rompre avec la théorie de la valeur-travail afin de " refonder le marxisme 62 ». Selon la perspective théorique de l'économie des conventions développée par Orléan, en dépit de son apparence hyper-rationnelle et hyper-abstraite, le domaine monétaire repose sur des normes sociales qui orientent les actions des acteurs ${ }^{63}$. L'évaluation financière est le

57 À ce sujet, voir Pierre Dusmenil, «Penser l'économie avec Castoriadis », Revue du Mauss, $\mathrm{n}^{\circ}$ 15, 2000, p. 171-185.

$5^{8}$ Cf. Serge Latouche, L'invention de l'économie, Paris, Albin Michel, 2011.

59 Cf. Bob Jessop et Ngai-Ling Sum, Towards a cultural political economy. Putting culture in its place in political economy, Cheltenham, Edward Elgar, 2014.

${ }^{60} \mathrm{Cf}$. Angus Cameron et Ronan Palan, The imagined economies of globalisation, London, Sage, 2004.

${ }^{61} \mathrm{Cf}$. André Orléan, L'empire de la valeur. Refonder l'économie, Paris, Seuil, 2011.

62 Ibid., p. 54.

63 Idem. 
produit d'une logique d'opinion autoréférentielle et l'institution de la bourse devient un lieu important de socialisation dans le capitalisme financiarisé. C'est l'opinion globale des investisseurs qui décide de la valeur des entreprises cotées en bourse et des taux d'emprunt des pays sur les marchés financiers. Dans cette logique d'opinion autoréférentielle, les acteurs n'agissent pas en fonction des critères de rationalité à la base des théories économiques néoclassiques, mais selon une rationalité mimétique. Fortement spéculatives, les décisions des investisseurs se prennent en anticipant constamment les croyances des autres. Cette spéculation se stabilise par des conventions d'interprétation, c'est-à-dire par des évaluations fondées sur une confiance dans le marché qui permet d'espérer que les revenus en termes de liquidités seront suffisamment élevés pour y justifier l'investissement ${ }^{64}$. Au contraire de la thèse néoclassique, la bourse n'est donc pas un lieu désocialisé où se rencontrent des acteurs rationnels et égoïstes. Elle est un lieu de socialisation dans lequel les acteurs réagissent fortement aux liens interpersonnels, aux rumeurs et aux modes 65 construites notamment par les agences de notation au cœur de l'actuelle crise financière mondiale ${ }^{66}$. Cette logique autoréférentielle repose sur la norme de rentabilité financière imposée par les gestionnaires de l'épargne collective. Le partage de l'épargne collective s'effectue par l'anticipation du cours des actions afin d'alimenter les profits. Cette dynamique autoréférentielle trouve ensuite écho dans la sphère productive sous la forme de la norme de la valeur actionnariale ${ }^{67}$.

Le système financier globalisé repose donc sur un nouvel imaginaire économique de la circulation ${ }^{68}$ qui vient remplacer l'imaginaire économique du capitalisme industriel fondé sur la production, selon les anthropologues LiPuma et Lee ${ }^{69}$. Dans ce nouvel imaginaire, le risque devient une nouvelle forme de médiation sociale fondée sur l'information financière. Le risque se substitue à la médiation des rapports sociaux via le travail abstrait. De la même manière dont l'échange d'une marchandise quelconque repose sur l'abstraction de sa spécificité et de sa qualité, la possibilité d'échanger des produits dérivés provient de l'abstraction de la spécificité d'une mul-

\footnotetext{
64 André Orléan, «L'individu, le marché et l'opinion : réflexions sur le capitalisme patrimonial », Esprit, vol. $11, \mathrm{n}^{\mathrm{o}} 269$, nov. 2000, p. 51-75.

$65 \mathrm{Idem}$.

${ }^{66}$ Cf. Timothy Sinclair, The new masters of capital american bond rating agencies and the politics of creditworthiness, Ithaca, Cornell University Press, 2005.

$67 C f$. Frédéric Lordon, Fonds de pension, piège à cons? Mirage de la démocratie actionnariale, Paris, Raisons d'agir, 2000, p. 68.

$68 C f$. Benjamin Lee and Edward LiPuma, "Cultures of circulation : the imaginations of modernity », Public Culture, vol. 14, n ${ }^{\circ}$ 1, p. 191-213.

69 Les anthropologues Benjamin Lee et Edward LiPuma, membres du Center for Transcultural Studies (CTS), mobilisent le concept d'imaginaire tel que développé par Charles Taylor. Pour une analyse de la comparaison entre les concepts de Taylor et de Castoriadis au sein du Center for Transcultural Studies (CTS), voir Dilip Parameshwar Gaonkar, "Toward new imaginaries : an introduction », Public Culture, vol. 14, n 1 1, 2002, p. 1-19.
} 
titude de risques particuliers qui, dans leur essence même, sont incommensurables ${ }^{70}$. Le risque abstrait est par la suite mesurable et décomposable en unités homogènes qui peuvent être échangées sur les marchés financiers sous la forme de produits dérivés ${ }^{71}$. La gestion du risque devient dès lors l'activité principale de la firme plutôt que la production de biens et services, puisque les risques peuvent être par la suite transformés en titres qui sont échangés sur les marchés financiers et sujets à la valorisation capitalistique. En clair, le risque objectivé sous la forme du produit dérivé est la principale marchandise échangée sur les marchés. Dans une économie financiarisée, la valeur des actifs des entreprises ne portent donc pas tant sur les produits et services qu'ils produisent que sur leur capacité de contrôle des risques.

En s'appuyant également sur l'analyse des fondements symboliques de l'échange marchand d'Aristote proposée par Castoriadis, Bichler et Nitzan soutiennent dans leur ouvrage Le capital comme pouvoir : une étude de l'ordre et du créordre ${ }^{72}$ que la valeur ne provient pas de la sphère matérielle de la consommation et de la production, mais plutôt des institutions sociales-juridiques-historiques plus larges de la société, ce que les Grecs appelaient le nomos. Le moteur du capitalisme repose sur un processus de capitalisation - c'est-à-dire l'actualisation des bénéfices futurs à la valeur actuelle. La puissance du capital se caractérise ainsi par sa possibilité d'imposer son nomos (sa loi), laquelle repose notamment sur sa capacité à quantifier l'inquantifiable afin de transformer l'ensemble de la vie sociale en flux de revenus qu'il est possible de s'accaparer. En ce sens, le processus d'accumulation du capital ne consiste pas en l'accumulation de richesse matérielle, mais plutôt en la «quantification symbolique du pouvoir73». Dans ce contexte, les capitalistes dominants (banques, investisseurs institutionnels, corporations) usent de diverses stratégies extraéconomiques afin de produire une accumulation différentielle qui consiste à restreindre l'accès aux flux de revenus à d'autres capitalistes subordonnés afin de dépasser le taux de rendement normal. Ces moyens sont les limitations directes, comme les prix d'éviction, les collusions formelles/informelles, la publicité et les contrats exclusifs ou encore des stratégies plus globales comme l'éducation ciblée, les lois sur les brevets et les droits d'auteur, les politiques industrielles, les régulations financières, une imposition à taux préférentiel, des monopoles légaux, la législation du travail, des accords ou des barrières de commerce et, bien sûr, soulignent Bichler et Nitzan, l'usage de la force, y compris militaire, pour réaliser des objectifs commerciaux différentiels 74 .

\footnotetext{
$70 \mathrm{Cf}$. Edward LiPuma et Benjamin Lee, « Financial derivatives and the rise of circulation », Economy and Society, vol. 34, $\mathrm{n}^{\circ}$ 3, 2005, p. 404-427.

${ }^{71} \mathrm{Idem}$.

${ }^{72} C f$. Jonathan Nitzan et Shimshon Bichler, Le capital comme pourvoir : une étude de l'ordre et du créordre, Paris, Max Milo, 2012.

73 Ibid., p. 26.

74 Ibid., p. 423.
} 


\section{Conclusion : ramener la politique dans la critique de l'économie politique}

Dans cet article, nous avons voulu montrer l'originalité de la critique castoriadienne de l'économie politique. Si elle s'appuie en partie sur l'analyse marxienne, on peut dire qu'elle s'en démarque sur un point particulier. En effet, on peut dire que la pertinence de l'analyse de l'imaginaire chez Castoriadis pour la sociologie en général, et plus particulièrement pour la sociologie critique, est qu'elle permet de dépasser certaines limites de l'approche marxienne, notamment la critique du fétichisme qui est, chez Marx, selon Jean-Louis Prat, "presque toujours synonyme d'illusion 75 ». En soulignant le rôle constitutif des imaginaires dans l'orientation significative des pratiques sociales, le principal apport de Castoriadis à la sociologie critique est de penser la nécessité des médiations institutionnelles et politiques contre ce qu'il nomme " la mauvaise utopie anarcho-marxiste [selon laquelle] un jour, les individus agiront spontanément de façon sociale et qu'il n'y aura besoin d'aucune contrainte, etc., et qu'il n'y aura même pas besoin de prendre de décisions collectives ${ }^{76} »$. De manière plutôt paradoxale, en sciences sociales, c'est au sein de la sociologie économique que les chercheurs mobilisent le concept d'institution d'un point de vue critique afin de dénaturaliser le marché. Pour la sociologie économique, le marché n'est pas une entité naturelle et transhistorique qui émergerait spontanément des échanges entre individus, il s'agit plutôt d'une institution social-historique qui, laissée à elle-même, mène à l'anéantissement de sa condition nécessaire de possibilité, c'est-à-dire la société77. A contrario, dans la majorité des travaux en sociologie critique, l'institution est généralement synonyme de domination illégitime. La tâche des sciences sociales «critiques" serait donc de démasquer les mécanismes du pouvoir institutionnel, principale source de l'oppression des individus. En découle une ontologie nominaliste et individualiste, hégémonique dans les sciences sociales contemporaines, lesquelles participent malgré elles à la reproduction de l'idéologie dominante ${ }^{78}$. En ce sens, l'apport principal de Castoriadis pour les sciences sociales, et la sociologie critique en particulier, est de nous rappeler qu'il ne peut exister de société sans institution.

\footnotetext{
75 Jean-Louis Prat, « Marx et l'imaginaire », Revue du Mauss, vol. 2, n 34, 2009, p. 394.

76 Cornelius Castoriadis, Démocratie et relativisme. Débats avec le Mauss, Paris, Mille et une nuits, 2010 , p. 46.

77 Voir à ce sujet Karl Polanyi, La Grande Transformation. Aux origines politiques et économiques de notre temps, Paris, Gallimard, 1983 [1944].

78 Christian Laval, «Le destin de l'institution dans les sciences sociales », Revue du MAUSS, vol. 2, $\mathrm{n}^{\mathrm{o}} 48,2016$, p. $275-292$.
} 